\title{
Current concepts to reduce mechanical stiffness in locked plating systems: a review article
}

\author{
This article was published in the following Dove Press journal: \\ Orthopedic Research and Reviews \\ 29 October 2014 \\ Number of times this article has been viewed
}

\author{
Nikhil Nanavati \\ Marcus Walker \\ Scunthorpe General Hospital, \\ Scunthorpe, North East \\ Lincolnshire, UK
}

Correspondence: Nikhil Nanavati Sheffield Teaching Hospital,

Sorby I Secretaries, Herries Road,

Sheffield, S5 7AU, UK

Tel +44 I724 282282

Email nikhilI0I@doctors.org.uk

\begin{abstract}
Locking plates can be used in a bridging mode to treat fractures by means of secondary bone healing. This requires stimulation by interfragmentary micromotion. Recent data suggest that up to $20 \%$ of fractures fixed with locking plates may encounter suboptimal fracture healing. Three main systems have been developed to improve bone healing, thereby reducing the risks of delayed or nonunion: overdrilling the near cortex; far cortical locking (FCL) screw; and dynamic locking screw (DLS). Overdrilling the near cortex offers a cheap alternative to screw technology by reducing construct stiffness; overdrilling potentially causes repetitive loading and thus has consequences postoperatively such as failure and fatigue. FCL screws reduce construct stiffness and maintain construct strength but cannot be used in dynamic compression mode and therefore, require adequate fracture reduction. DLS is a new generation of locking screw, amalgamating locking technology with dynamic motion with the aim of improving interfragmentary motion at the near cortex.
\end{abstract}

Keywords: dynamic locking screw, locking plates, bone healing, construct stiffness, dynamic compression

\section{Introduction}

Recent data suggest that up to $20 \%$ of fractures fixed with locking plates may encounter suboptimal fracture healing. ${ }^{1}$ Locking plate systems have several advantages over conventional screw systems. A conventional plate or screw system requires the plate to be adapted precisely to the underlying bone. This need for the closeness of the plate to the bone means that tightening of screws results in different alignments between the osseous segments. Locking plates do not need to have this close contact to the bone due to the screws locking the plate and thus the plate stabilizes the bone without the need for the plate to compress the bone and therefore the alignment of the osseous segments has better chance of being maintained. This system of not having the bone compressed to the plate means that cortical bone perfusion is not disrupted as much. ${ }^{2}$ Strain theory is where a material deforms when a given force is applied. Normal strain is the change in length in comparison to original length when a given load is applied. ${ }^{3}$ This is worth bearing in mind with the application of locking plates.

Locking plates can be used in a bridging mode to treat fractures by means of secondary bone healing. This requires stimulation by interfragmentary micromotion. By allowing the screw heads to engage away from the bone surface, these plates reduce disruption of the periosteum and blood supply. They are particularly useful for fractures in osteoporotic bone where it can be difficult to obtain a good hold, which can result in 
loosening of a fixation. The working length of a locking plate primarily influences axial stiffness. The working length is defined as the two nearest points where the bone is fixed to the plate. Screws placed close to the fracture site have a reduced working length and increase the stiffness of the construct.

Locking plates can fatigue or fail in long unsupported sections or if it is stressed too much at one level. Locking plate failure can occur with periprosthetic fracture. If all the locking screws are utilized a stiff construct is created, this puts a very sharp transition of stress at the end of the implant increasing the risk of periprosthetic fracture. ${ }^{4}$ Poor bone quality can also lead to a locking plate failing due to the screws not having purchase. ${ }^{5}$

By aiming for a plate length that is greater than two or three times the working length of the fracture and a screw density ratio of 0.4 to 0.5 , the operator is able to fill fewer than half of the holes with screws and prevent the construct becoming too stiff and developing nonunion (as the working length is inversely proportional to the stiffness).

Inversely screw plates further away from the fracture have an increased working length and decrease the stiffness of the construct. By increasing the working length of the plate and reducing the number of screws used in the construct, it is possible to reduce the stiffness at the far cortex but not at the near cortex. ${ }^{6}$

Locking plates have a fixed connection between threads in the head of the screws and the plate. During axial compression, this construct displays greater rigidity at the near cortex of the fracture site and less rigidity at the far cortex, where bending can occur. This therefore means that more micromotion occurs at the far cortex compared to the near, stimulating bone healing at the far cortex by means of indirect bone healing through callus formation. ${ }^{7}$ If the construct is too rigid, micromotion is unable to occur at both the near and far cortices at the fracture site, thereby inhibiting bone healing. Biomechanical studies ${ }^{7,8}$ into the rigidity of locking plates have shown that there is minimal interfragmentary motion at the near cortex and much greater at the far cortex. ${ }^{8}$ Thus, postoperative interfragmentary micromotion during weight bearing may be too insignificant to achieve callus formation when using locking plates.

Due to concerns regarding reduced micromotion at fracture sites with locking plates, various systems have been developed to improve bone healing, thereby reducing the risks of delayed or nonunion. The aim of this literature review is to evaluate the outcome of various modern systems on bone healing through a detailed review of the available literature.

\section{Overdrilling the near cortex}

When using a locking plate, it is possible to overdrill the near cortex to allow a degree of micromotion to stimulate fracture healing. Gardner et $\mathrm{a}^{10}$ used synthetic bone to create a fracture gap with the application of a locking plate. To guarantee anchorage of the plate it is necessary for screw threads to engage in the bone cortex over a sufficient distance. A normal cortex has enough thickness to allow adequate anchorage. In osteoporosis the cortex is thinner which reduces the working length of the screw which leads to an increased risk of failure. Thus in osteoporosis a low energy level can damage the screw bone interface with subsequent loosening and instability. Therefore in osteoporosis it necessary to use bicortical screws rather than a monocortical screw. ${ }^{9}$

Each of the two fragments had four bicortical locking screws applied, however, in one of the fragments, the near cortices were milled to $5 \times 6 \mathrm{~mm}$ slots in increments (ie, $0-4$ of the holes) to allow for translation of the screw shaft. ${ }^{10}$

Axial stiffness of the milled four-hole configuration was found to have decreased by $73 \%$, whilst torsional stiffness decreased by $17 \%$ when compared with the zero-hole configuration. The study also identified that there was no effect on the variable constructs after cyclical loading and fixation was maintained.

Osteoporotic bone has been shown to decrease axial stiffness, however, due to the mismatch between the high axial stiffness of a plate there is risk of nonunion, failure, or cut-out of the screw-plate construct. ${ }^{11,12}$

Gardner et al used a substitute osteoporotic bone specimen to fix a locking plate in two various ways: one with slots instead of holes and one with standard holes at the near cortices. ${ }^{13}$

The study identified that it is possible to create a more stable construct by using the near slotted hole configuration. The stiffness of the slotted group was found to be one third of the non-slotted group on cyclical loading. It had already been identified that axial stiffness greater than 2,400 N/mm leads to impaired bone healing and most locking plates have a value higher than this. ${ }^{14}$ Gardner's study identified that by using near cortical slots, axial stiffness is reduced to below this threshold.

Chen et al devised the concept of drilling "figure of 8 " holes in the near cortices to reduced axial stiffness. ${ }^{15}$ Synthetic bone with fracture gaps was fixed with locking plates in a six hole bicortical screw configuration. The test group had the near cortices drilled in a "figure of 8" pattern using a $4.3 \mathrm{~mm}$ drill bit. 
It was found that axial stiffness was $16.07 \%$ less in the test group ("figure of 8") when loaded in axial compression. However, this study also identified that axial stiffness was significantly reduced after cyclical loading from $3,000<x<10,000$ (with $\mathrm{x}$ being the number of cycles). By interpreting these results, the "figure of 8 " holes can be a cost-effective measure in reducing axial stiffness without affecting the strength of the construct. This study went on to conclude that patients should only be partially weight bearing following this method of fixation due to the diminished stiffness on repetitive loading.

\section{Far cortical locking}

Far cortical locking (FCL) is an approach requiring specialized screws in a locking plate. FCL aims to reduce stiffness whilst maintaining strength of a construct. The screws have threads at the tip and the head that lock into the plate and far cortex respectively. The screws also have a shaft with a reduced diameter, allowing for movement at the near cortex due to an increased working length.

In order to ensure that screws are lined up correctly with axis of the screw threads in the plate they need to be inserted in the plate holes. Following this the operator picks the appropriate drill sleeve and threads it into the locking insert to achieve initial fixation of the plate.

The operator then inserts the correct sized locking screw. Locking screws should initially only be placed manually to guarantee alignment. If the locking screw thread is not engaged immediately to the plate thread, the operator needs to reverse the screw several turns and reinsert the screw once it is aligned correctly.

FCL screws have been shown to reduce the axial stiffness of: 1) metaphyseal femoral fractures fixed with locking plates by $80 \% ;{ }^{8}$ 2) femoral diaphyseal fractures fixed with a standard locked plating system by $88 \%,{ }^{16}$ 3) tibial diaphyseal fractures fixed with a locking plate in bridging mode by $84 \% .{ }^{17}$

This reduced stiffness allows for similar biomechanical principles involved in external pin fixation to be applied, thereby allowing interfragmentary motion to induce secondary bone healing.

As mentioned previously, gap healing can occur with standard locking plates. ${ }^{10}$ Bottlang et al carried out tests on locking plate constructs and FCL constructs in a diaphyseal bridge plating model of nonosteoporotic femoral diaphysis; they also tested the constructs to failure ${ }^{16}$ However, plates with FCL screws show evidence of improved parallel fracture site interfragmentary motion. ${ }^{16}$ Bottlang's study found that at $200 \mathrm{~N}$ weight-bearing, a fracture fixed with an FCL construct had gap motion of $0.59 \mathrm{~mm}$ at the far cortex and $0.51 \mathrm{~mm}$ at the near cortex. ${ }^{16}$ The standard (control) locked construct only had a gap motion of $0.05 \mathrm{~mm}$ at the far cortex and $0.02 \mathrm{~mm}$ at the near cortex. Bottlang et al determined that a FCL construct is $54 \%$ stronger in torsion and $21 \%$ have greater resistance to bending moment when compared with a standard locking plate construct. ${ }^{16}$

FCL constructs form more callus and promote fracture healing more significantly than standard locking plate constructs. ${ }^{21}$ Tibial osteotomies were performed in 12 sheep randomly assigned for fixation with FCL or a standard locking construct.

Weekly radiographs were performed for 9 weeks after which time the sheep were sacrificed and the tibiae were further visually (computed tomography and histology) analyzed and mechanically tested (torsional strength). The FCL group was found to have consistently developed significantly more callus which was more evenly distributed around the cortices, whilst half (3/6) of the control group developed a partial nonunion. The FCL group was also found to be $54 \%$ stronger and withstood over three times the amount of energy before failing compared with the control group. ${ }^{17}$

\section{Dynamic locking screw}

The dynamic locking screw (DLS) is a new generation of locking screw that reduces construct stiffness and minimizes soft tissue damage. DLS is formed from two key components; firstly, the outer sleeve engaging within bone (cancellous and far cortical) and secondly, the inner pin that lies within the outer sleeve and locks into the plate. The interaction between the two elements allows the DLS to utilize locking technology and amalgamates it with dynamic motion, thereby enhancing interfragmentary motion at the near cortex.

Doebele et al used a validated, optical, three-dimensional measurement system (PONTOS 5M) to study micromotion at the fracture site using dynamic locking screws. The study created three kinds of fracture patterns (transverse, oblique and spiral) each with a fracture gap of $3 \mathrm{~mm}$. Standardized configurations of a locking plate with dynamic locking screws or standard locking screws were assessed for axial stiffness and micromotion by applying an axial load of increasing force (0-200 N). From a total of 72 constructs, axial stiffness was found to have reduced by $16 \%$ when using the DLS and micromotion was found to have increased by $141 \mu \mathrm{m}$ (from 282 to $423 \mu \mathrm{m}$ ). ${ }^{18}$

Plecko et al designed an in vivo study looking at the outcomes of fracture fixation with DLS in sheep. ${ }^{19} \mathrm{~A}$ total of 36 sheep were divided into three groups with varying $45^{\circ}$ osteotomy gaps performed; $0 \mathrm{~mm}, 1 \mathrm{~mm}$, and $3 \mathrm{~mm} .{ }^{19}$ 
The sheep were radiologically assessed every 3 weeks and were sacrificed at either 6 or 12 weeks allowing for biomechanical testing and further radiological assessment.

The group with a $0 \mathrm{~mm}$ fracture gap was found to actually have greater callus formation at the near cortex (36.44\%) compared with the far $(32.09 \%)(P=0.078)$. In addition, they also displayed greater callus stiffness at earlier healing-time points when compared to the other groups.

It can therefore be proposed that by reducing fracture fragments appropriately and using a locking plate with DLS, one can induce greater callus stiffness and more symmetrical callus formation in certain fracture patterns.

At the time of writing, a multicenter randomized control study assessing the outcomes of fixation of distal femur fractures with standard locking screws and dynamic locking screws is currently being performed in Washington, USA. The study aims to assess postoperative pain, stiffness and physical function of patients using a WOMAC (Western Ontario and McMaster Universities Index) score.

A further study has also been proposed at Berufsgenossenschaftliche Unfallklinik Hospital, Tübingen, Germany assessing micromotion in cadaveric spines with thoracolumbar fractures fixed with DLS. The study is aiming to show that DLS can theoretically prevent delayed bone healing by using three-dimensional optical analysis to assess vertical micromotion between two segments. Biomechanical testing will also be performed to assess torsional stability and flexibility of the DLS/plate screw construct.

\section{Conclusion}

Locking plate technology has revolutionized options for fracture fixation, especially with regards to minimizing trauma to soft tissues and periosteum, thereby promoting osteosynthesis.

Whilst construct rigidity offers benefits from mechanical strength, it may also lead to delayed bone healing due to suppression of interfragmentary micromotion required for callus formation. It has already been shown that motion in order of $400 \mu \mathrm{m}$ at the near cortex leads to improved callus formation and various concepts have been developed to reduce axial stiffness whilst maintaining construct strength to allow for this. ${ }^{10,20}$

Overdrilling the near cortex offers a cheap alternative to screw technology by reducing construct stiffness; however this may prove to be significant on repetitive loading and may therefore have consequences postoperatively. This method is also user-dependent and whilst many overdrilling techniques have been used, none have actually measured the amount of motion at the fracture gap.
Far cortical locking screws have proven benefits with regards to improving callus formation, reducing construct stiffness and maintaining construct strength. However, these screws cannot be used in a dynamic compression mode and require the operator to ensure adequate fracture reduction by other means. ${ }^{21}$

The DLS is a relatively new concept with emerging evidence to display its benefits. Whilst the screws are relatively expensive, they offer the operator the option of using them in compression mode, thereby reducing the fracture gap and promoting improved healing by reducing strain across the fracture gap. However, due to its recent introduction, there is currently not enough evidence to establish whether the DLS has a proven role in fracture management.

To date, there is no research comparing axial stiffness, torsional stiffness, construct strength (on repetitive loading) and interfragmentary micromotion in a standardized fracture pattern using each of the various techniques mentioned in this article. This is an area for further research.

\section{Disclosure}

The authors report no conflicts of interest in this work.

\section{References}

1. Henderson CE, Bottlang M, Marsh JL, Fitzpatrick DC, Madey SM. Does locked plating of periprosthetic supracondylar femur fractures promote bone healing by callus formation? Two cases with opposite outcomes. Iowa Orthopaedic Journal. 2008;28:73-76.

2. Locking versus nonlocking plates - Advantages to a locking plate/ screw system [webpage on the Internet]. Davos: AO Foundation; 2014. Available from: https://www2.aofoundation.org/wps/portal/!ut/p/c0/04_ SB8K8xLLM9MSSzPy8xBz9CP0os3hng7BARydDRwN39yBTAyMvLwOLUA93I4MQE_2CbEdFAF3RnT4!/?contentUrl=\%2Fsrg\%2Fpo pup $\% 2$ Fadditional_material\%2F91\%2FX40_Lockplate_principles.jsp $\&$ popupStyle $=$ diagnosis $\&$ soloState $=$ true $\&$ bone $=\mathrm{CMF} \&$ segment $=\mathrm{Man}$ dible \&BackMode=true. Accessed June 2, 2014.

3. Perren SM, Cordey J. The Concept of Interfragmentary Strain. New York: Springer; 1980.

4. Maureen Leahy. American Academy of Orthopaedic Surgeons (AAOS). Webpage on the Internet. When locking plates fail. Available from: http://www.aaos.org/news/aaosnow/may10/clinical4.asp. Accessed June $2,2014$.

5. Walter W Virkus, Benjamin J Miller, Ping C Chye, Steven Gitelis. The Use of Locking Plates in Orthopedic Oncology Reconstructions Orthopedics. May 2008;31(5).

6. Stoffel K, Dieter U, Stachowiak G, Gächter A, Kuster MS. Biomechanical testing of the LCP - how can stability in locked internal fixators be controlled? Injury. 2003;34(Suppl 2):B11-B19.

7. Unthoff H, Poitras P, Backman DS. Internal plate fixation of fractures: short history and recent developments. J Orthop Sci. 2006;11(2): $118-126$.

8. Fitzpatrick DC, Doornink J, Madey SM, Bottlang M. Relative stability of locked plate fixation in a model of the osteoporotic femoral diaphysis. Clin Biomech. 2009;24(2):203-209.

9. http://issuu.com/aofoundation/docs/aodialogue_2009_02_14. Bridge plating Expert zone Clinical topic, by Emanuel Gautier, p24 accessed 2-6-14. 
10. Gardner MJ, Nork SE, Huber P, Krieg JC. Stiffness Modulation of Locking Plate Constructs Using Near Cortical Slotted Holes: A Preliminary Study. J Orthop Trauma. 2009;23(4):281-287.

11. Burstein AH, Reilly DT, Martens M. Aging of bone tissue: mechanical properties. J Bone Joint Surg Am. 1976;58:82-86.

12. Bottlang M, Doornink J, Byrd GD, Fitzpatrick DC, Madey SM. A nonlocking end screw can decrease fracture risk caused by locked plating in the osteoporotic diaphysis. J Bone Joint Surg Am. 2009;91: 620-627.

13. Gardner MJ, Nork SE, Huber P, Krieg JC. Less rigid stable fracture fixation in osteoporotic bone using locked plates with near cortical slots. Injury. 2010;41:652-656.

14. Epari DR, Kassi JP, Schell H, Duda GN. Timely fracture-healing requires optimization of axial fixation stability. J Bone Joint Surg Am. 2007;89:1575-1585.

15. Chen J, Zhihong Z, Hong B, et al. Can Over-drilling the Near Cortex Reduce the Stiffness of Locking Plate-bone Constructs? Am Academy Orth Surg. 2013;631-645.
16. Bottlang M, Doornink J, Fitzpatrick DC, Madey SM. Far Cortical Locking can reduce the stiffness of locked plating constructs while retaining construct strength. J Bone Joint Surg Am. 2009;91:1985-1994.

17. Bottlang M, Lesser M, Koerber J, et al. Far cortical locking can improve healing of fractures stabilized with locking plates. J Bone Joint Surg Am. 2010;92:1652-1660.

18. Doebele S, Horn C, Eichhorn S, Lucke M, Koch R, Stöckle U. Dynamic Locking Screws (DLS) - Biomechanical testing of a new screw system. J Bone Joint Surg Br. 2011;93(Suppl II):100.

19. Plecko M, Lagerpusch N, Andermatt D, et al. The dynamisation of locking plate osteosynthesis by means of dynamic locking screws (DLS) - An experimental study in sheep. Injury. 2013;44(10): 1346-1357.

20. Wolf S, Janousek A, Pfeil J, et al. The effects of external mechanical stimulation on the healing of diaphyseal osteotomies fixed by flexible external fixation. Clin Biomech. 1998;13:359-336.

21. Bottlang M, Feist F. Biomechanics of Far Cortical Locking. J Orthop Trauma. 2011;25:21-28.
Orthopedic Research and Reviews

\section{Publish your work in this journal}

Orthopedic Research and Reviews is an international, peer-reviewed, open access journal focusing on the patho-physiology of the musculoskeletal system, trauma, surgery and other corrective interventions to restore mobility and function. Advances in new technologies, materials, techniques and pharmacological agents are particularly welcome. The journal welcomes

\section{Dovepress}

original research, clinical studies, reviews \& evaluations, expert opinion and commentary, case reports and extended reports. The manuscript management system is completely online and includes a very quick and fair peer-review system, which is all easy to use. Visit http://www.dovepress. com/testimonials.php to read real quotes from published authors.

Submit your manuscript here: http://www.dovepress.com/orthopedic-research-and-reviews-journal 